\title{
Smad4 sensitizes colorectal cancer to 5-fluorouracil through cell cycle arrest by inhibiting the PI3K/Akt/CDC2/survivin cascade
}

\author{
BINHAO ZHANG, CHAO LENG, CHAO WU, ZHANGUO ZHANG, LEI DOU, \\ XIN LUO, BIXIANG ZHANG and XIAOPING CHEN \\ Hepatic Surgery Center, Tongji Hospital, Tongji Medical College, Huazhong University \\ of Science and Technology, Wuhan, Hubei 430030, P.R. China
}

Received September 24, 2015; Accepted December 17, 2015

DOI: $10.3892 /$ or.2015.4479

\begin{abstract}
Fluorouracil (5-FU), a cell cycle-specific antimetabolite, is one of the most commonly used chemotherapeutic agents for colorectal cancer (CRC). Yet, resistance to 5-FU-based chemotherapy is still an obstacle to the treatment of this malignancy. Mutation or loss of Smad4 in CRC is pivotal for chemoresistance. However, the mechanism by which Smad4 regulates the chemosensitivity of CRC remains unclear. In the present study, we investigated the role of Smad4 in the chemosensitivity of CRC to 5-FU, and whether Smad4-regulated cell cycle arrest is involved in 5-FU chemoresistance. We used Smad4-expressing CT26 and Smad4-null SW620 cell lines as experimental models, by knockdown or transgenic overexpression. Cells or tumors were treated with 5-FU to determine chemosensitivity by cell growth, tumorigenicity assay and a mouse model. Cell cycle distribution was examined with flow cytometric analysis, and cell cycle-related proteins were examined by western blotting. Smad 4 deficiency in CT26 and SW620 cells induced chemoresistance to 5-FU both in vitro and in vivo. Smad4 deficiency attenuated G1 or G2 cell cycle arrest by activating the PI3K/Akt/CDC2/survivin pathway. The PI3K inhibitor, LY294002, reversed the activation of the Akt/CDC2/survivin cascade in the Smad4-deficient cells, while it had little effect on cells with high Smad4 expression. In conclusion, we discovered a novel mechanism mediated by Smad4 to trigger 5-FU chemosensitivity through cell cycle arrest by inhibiting the PI3K/Akt/CDC2/survivin cascade. The present study also implies that LY294002 has potential therapeutic value to reverse the chemosensitivity of CRC with low Smad4 expression.
\end{abstract}

Correspondence to: Professor Xiaoping Chen or Dr Binhao Zhang, Hepatic Surgery Center, Tongji Hospital, Tongji Medical College, Huazhong University of Science and Technology, 1095 Jie Fang Avenue, Wuhan, Hubei 430030, P.R. China

E-mail: chenxp@medmail.com.cn

E-mail: bhzhang8@163.com

Abbreviations: 5-FU, 5-fluorouracil; CRC, colorectal cancer

Key words: colorectal cancer, 5-fluorouracil, chemotherapy, cell cycle, Smad4

\section{Introduction}

Colorectal cancer (CRC) is one of the most prevalent malignancies in China and many other parts of the world. The American Cancer Society recently reported that the rate of incidence of and death due to CRC have undergone a rapid decline in the past 30 years, owing to the improvement in early detection and/or treatment (1). However, personalized therapeutic strategies targeting different pro-oncogenic factors and overcoming drug resistance are still issues in CRC treatment.

Previous clinical research indicated that Smad4 is a predictive biomarker for 5-fluorouracil (5-FU)-based chemotherapy in patients with CRC (2-6). In addition, a recent in vitro study provided evidence that Smad4 inactivation promotes the resistance of CRC to 5-FU treatment and hypoxiainduced cell death (7). Low Smad4 expression in human CRC predicts early recurrence after curative therapy (4), less response to 5-FU $(2,3)$ and shorter overall and disease-free survival time $(2,3,8)$. However, the mechanism concerning the promotive effect of Smad4 on drug sensitivity remains to be elucidated. The PI3K/Akt pathway has been shown to play an important role in promoting cell survival and inhibiting apoptosis. Owing to these effects, the PI3K/Akt pathway may affect cellular responses to DNA damage and cell cycle checkpoints induced by DNA damage. As crosstalk between Smad and the non-Smad pathway exists, modification of the non-Smad pathway by Smad4, including the PI3K/Akt pathway, may effect drug sensitivity.

The present study suggests, for the first time, that Smad4 has an effect on the induction of chemosensitivity both in murine CT26 cells and human SW620 cells, through G1 or $\mathrm{G} 2$ cell cycle arrest by inhibiting the PI3K/Akt/CDC2/ survivin pathway. LY294002 reversed the chemosensitivity of CRC with low Smad4 expression by inhibiting the PI3K/ Akt/CDC2/survivin cascade. These findings imply that Smad4 may be a candidate biomarker for the combined use of LY294002 and 5-FU-based chemotherapy for patients with CRC.

\section{Materials and methods}

Cell lines, animals, reagents and antibodies. CT26 cells, an undifferentiated murine adenocarcinoma-derived cell line 
from the BALB/c mouse, and human colon adenocarcinoma cell line SW620 were maintained in RPMI-1640 containing $10 \%$ fetal bovine serum (FBS). Smad4 knockdown CT26 cells and SW620 cells expressing Smad4 were established as previously described (9). Female BALB/c and athymic nude mice ( 6 weeks of age) were used for the experiments. All animal studies were approved by the Ethics Committee of Huazhong University of Science and Technology. 5-FU was obtained from Sigma. The PI3K inhibitor, LY294002, and the MEK1/2 inhibitor, PD98059, were obtained from Calbiochem (San Diego, CA, USA). Antibodies were purchased as follows: anti-Smad4, anti-cyclin B1, anti-survivin, anti-CDC2, antiERK (Santa Cruz Biotechnology, Santa Cruz, CA, USA); anti-cleaved-caspase 3, anti-p-ERK, ant-p-Akt and anti-Akt (Cell Signaling Technology, Inc., Danvers, MA, USA).

Cell counting assay. CT26 cells (2,000/well) or SW620 cells (3,000/well) were seeded into 12-well plates and were cultured in media with $10 \%$ FBS. Media were replaced every other day. Cells were counted each day, and the average cell numbers from triplicate measurements were plotted.

Cell isolation from tumor tissues. The liver metastasis model was established by splenic injection of CT26 cells in BALB/c mice as previously described (10). Liver metastases were removed 4 weeks after cell inoculation and cut into small pieces in phosphate-buffered saline (PBS). Tissues were then incubated with collagenase (Sigma) medium for 3-4 h at $37^{\circ} \mathrm{C}$, under shaking. Cells were centrifuged and washed with Dulbecco's modified Eagle's medium (DMEM) 4-5 times, and then suspended in complete DMEM in $10 \mathrm{ml}$ for $2 \mathrm{~min}$. Eight milliliters was taken from the top for culture, and the liver metastasis cell line (CT26 LM1) was established. LM1 cells were splenic injected into the mice, and LM2 cells were established by repeating the experiment.

Soft agarose tumorigenicity assay. Semisolid (1 ml) $0.8 \%$ sea plaque agarose was plated into 6-well plates, allowed to solidify for at least $1 \mathrm{~h}$. Then, CT26 $\left(1.0 \times 10^{4}\right)$ and SW620 $\left(2.5 \times 10^{4}\right)$ cells were suspended in $1 \mathrm{ml}$ of $0.4 \%$ agarose containing $7 \%$ FBS and plated on the top of the agarose. After $48 \mathrm{~h}$, the cells were treated with media containing $20 \mu \mathrm{M}$ 5-FU. The media were replaced with or without 5-FU every other day. Images of the colonies were captured by phase contrast microscope 10 days after cell splitting.

Western blotting. Lysates from the CT26 or SW620 cells were treated as indicated, and tumors from the animal models were used for western blot analysis as previously described (11).

Flow cytometric analysis for cell cycle analysis. Cell cycle distribution analysis was performed by flow cytometry as previously described (12). Cells (1x105/well) were split into 6 -well plates and were incubated in serum-free media for $48 \mathrm{~h}$ to synchronize the cell cycle. Then cells were treated with kinase inhibitors and/or different concentrations of 5-FU for 48 h. Cells were harvested, washed twice with PBS and were fixed in $70 \%$ cold ethanol, and stained with propidium iodide (PI) in PBS containing RNase (both from Sigma). Stained cells were analyzed.
Tumorigenicity assay. CT26 $\left(1 \times 10^{5}\right)$ or SW620 cells $\left(2 \times 10^{6}\right)$ were injected subcutaneously into BALB/c or athymic nude mice $(n=5)$, respectively. 5-FU treatment was started as soon as the tumor had reached palpable size. For the SW620 tumor xenografts, 5 -FU $(50 \mathrm{mg} / \mathrm{kg})$ was administered i.p. on a 3 times/week basis for three consecutive weeks, and for CT26 allografts, 5-FU (100 mg/kg) was administered once a week during the first two weeks and $50 \mathrm{mg} / \mathrm{kg}$ once a week thereafter as previously described $(13,14)$. Tumor volume was calculated as previously described (10). Mice were sacrificed according to IACUC guidelines.

MTT cell proliferation assay and $I_{50}$ calculation. CT26 and SW620 cells $\left(5 \times 10^{3}\right)$ were plated into 96-well flat-bottomed microtiter plates and were incubated overnight. Cells were treated with the indicated concentrations of 5-FU for $72 \mathrm{~h}$. The number of viable cells was determined by MTT assay following the kit protocol (CT01; Millipore). The percent viability was calculated as: [Absorbance of drug-treatment)/ (control absorbance)] x $100 \% . \mathrm{IC}_{50}$ values were calculated using linear or logarithmic regression $\left(\mathrm{R}^{2}>0.9\right)$.

Cell migration assay. Cells $\left(2 \times 10^{4}\right)$ in $100 \mu 1$ serum-free media were seeded to the upper chamber of $8-\mu \mathrm{m}$ pore Transwells coated with collagen. Cells were allowed to migrate towards $10 \%$ FBS containing medium in the lower chamber for $5 \mathrm{~h}$. Cells on the upper side of the membrane were removed with a cotton swab. Cells that passed through the Transwells were fixed with $4 \%$ paraformaldehyde and stained with $1 \%$ crystal violet. Cells in five random fields (magnification, $x 200$ ) in each well were counted.

Statistical analysis. The Student's t-test was used to determine the statistical differences among the analyzed groups. Calculations were performed using Prism 5.0 software for Macintosh (GraphPad Software, San Diego, CA, USA). P<0.05 was considered to indicate a statistically significant result.

\section{Results}

Highly malignant CRC cells exhibit lower Smad4 expression. A highly aggressive and highly metastatic mouse cell line was generated using a splenic injection model of liver metastasis. CT26 cells have less potential to produce liver metastasis. To develop this highly metastatic cell line, CT26 cells were injected into the spleen of BALB/c mice, and 4 weeks later the mice were sacrificed, liver metastases were harvested and the cell line was established. Following this procedure, a highly metastatic CT26-LM2 cell line was established after 2 cycles of stepwise selection (Fig. 1A). The aggressiveness of the LM2 cells was tested by in vitro migration assays using Boyden chambers. We found that the LM2 cells exhibited a 2.7-fold increase in the level of cell migration as compared to the CT26 parental cells (Fig. 1B). Therefore, these results confirmed that the LM2 cells are highly aggressive. We next examined the Smad4 expression in the CT26 parental and LM2 cells. Smad4 expression in the LM2 cells was sharply lower when compared with the parental CT26 cells (Fig. 1C). These results indicated that lower Smad4 expression predicts higher malignancy in CRC. 
A

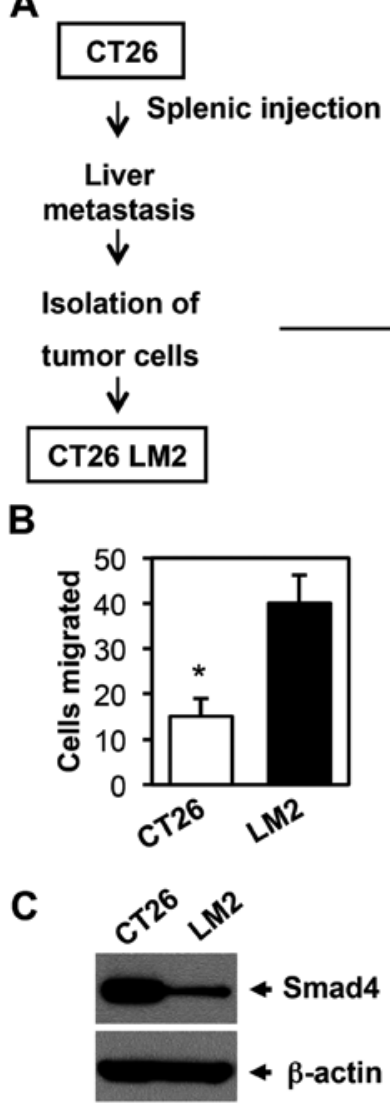

D

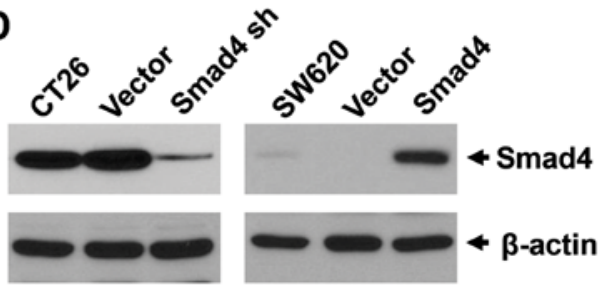

E

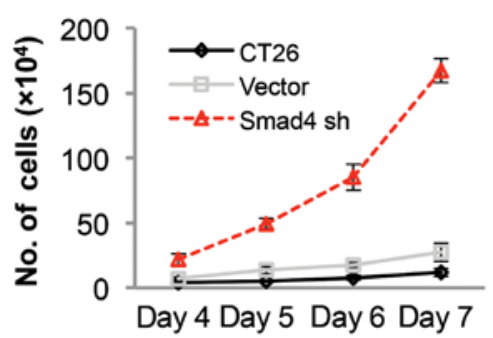

$\mathbf{F}$

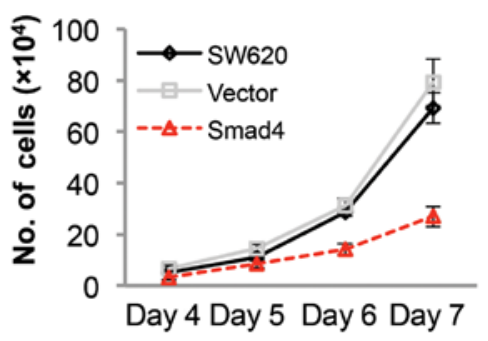

Figure 1. Smad4 reduces tumor malignancy in CRC cells. (A) Liver metastatic CT26 cell line LM2 was established by in vivo selection for two cycles. (B) Cell migration assay. Parental CT26 and LM2 cells were allowed to migrate through 8- $\mu$ m pore Transwells and pass through a collagen layer in the Transwell chamber. Migratory cells in five random fields in each well were counted. Each data point represents the mean \pm SD number from three wells. (C and D) Smad4 expression in CT26 parental and LM2 cells (C), and in Smad4-knockdown CT26 cells (Smad4 sh) and Smad4-overexpressing SW620 cells (Smad4) (D) was tested by western blotting. (E and F) Cell counting assays. CT26 and SW620 cells were cultured in 12-well plates with 7\% FBS for 7 days, and cells were counted every day. Each data point represents the mean \pm SD number from three wells. ${ }^{*} \mathrm{P}<0.001$.

Smad4 inhibits the proliferation of $C R C$. In order to investigate the role of Smad4 in CRC progression and drug sensitivity, we established a Smad4-knockdown mouse CRC cell line (CT26 Smad4 sh) and a Smad4-overexpressed human CRC cell line (SW620 Smad4). Smad4 expression was examined by western blotting (Fig. 1D). Cell proliferation was assessed by a cell counting assay. Smad4 deficiency significantly promoted cell proliferation in the CT26 cells (Fig. 1E), while Smad4 overexpression reduced the cell proliferation in the SW620 cells (Fig. 1F). In summary, Smad4 reduces cell proliferation in $\mathrm{CRC}$, which is consistent with our previous study (9).

Smad4 promotes the chemosensitivity of CRC to 5-FU in vitro and in vivo. To determine whether Smad4 affects the chemosensitivity of CRC cells, we first treated the CRC cells with 5-FU. After a 48-h treatment with 5-FU, the CT26 empty vector group presented a large number of floating cells, while the Smad4-knockdown group showed few floating cells (Fig. 2A). Consistent with this result, 5-FU killed more cells in the Smad4-overexpressing SW620 cells when compared with that in the empty vector group (Fig. 2B). We next treated the CRC colonies in soft argarose with 5-FU, and achieved the confirmed result (Fig. 2C and D). MTT cell proliferation assay was applied to check the $\mathrm{IC}_{50}$ value to $5-\mathrm{FU}$ in the different cells. The $\mathrm{IC}_{50}$ value to $5-\mathrm{FU}$ in the
CT26 Smad4-knockdown group was $>5$ times higher when compared with the CT26 vector control group (Fig. 2E). In contrast, in the Smad4-overexpressing SW620 cells, the $\mathrm{IC}_{50}$ value of 5-FU was $>2.6$ times lower when compared with the $\mathrm{IC}_{50}$ value in the vector control group (Fig. 2F).

We next treated the mice bearing CRC tumors with 5-FU to investigate the effect of Smad4 on 5-FU drug sensitivity in vivo. At the end of the experiment, the mice were sacrificed according to the IACUC standard and tumors were removed and measured. 5-FU reduced the tumor volume by 4.2 times in the CT26 empty vector group, while it reduced the tumor volume by only 0.2 times in the CT26 Smad4-knockdown group (Fig. 3A). In the SW620 empty vector control group, the tumor volume was reduced only by 0.2 times following 5-FU treatment. In the SW620 Smad4-overexpressing group, the tumor volume was reduced by 5.9 times following 5-FU treatment (Fig. 3B). These results provide evidence that $\mathrm{Smad} 4$ promotes chemosensitivity to 5-FU in CRC.

Smad4 deficiency in CRC activates Akt and ERK, and attenuates cell cycle arrest in the G1 and G2 phases. As crosstalk between Smad and the non-Smad pathway exists, and several non-Smad pathways were reported to affect chemosensitivity, we examined the activation of Akt and ERK in the Smad4-knockdown or overexpressing cells. Consistent 

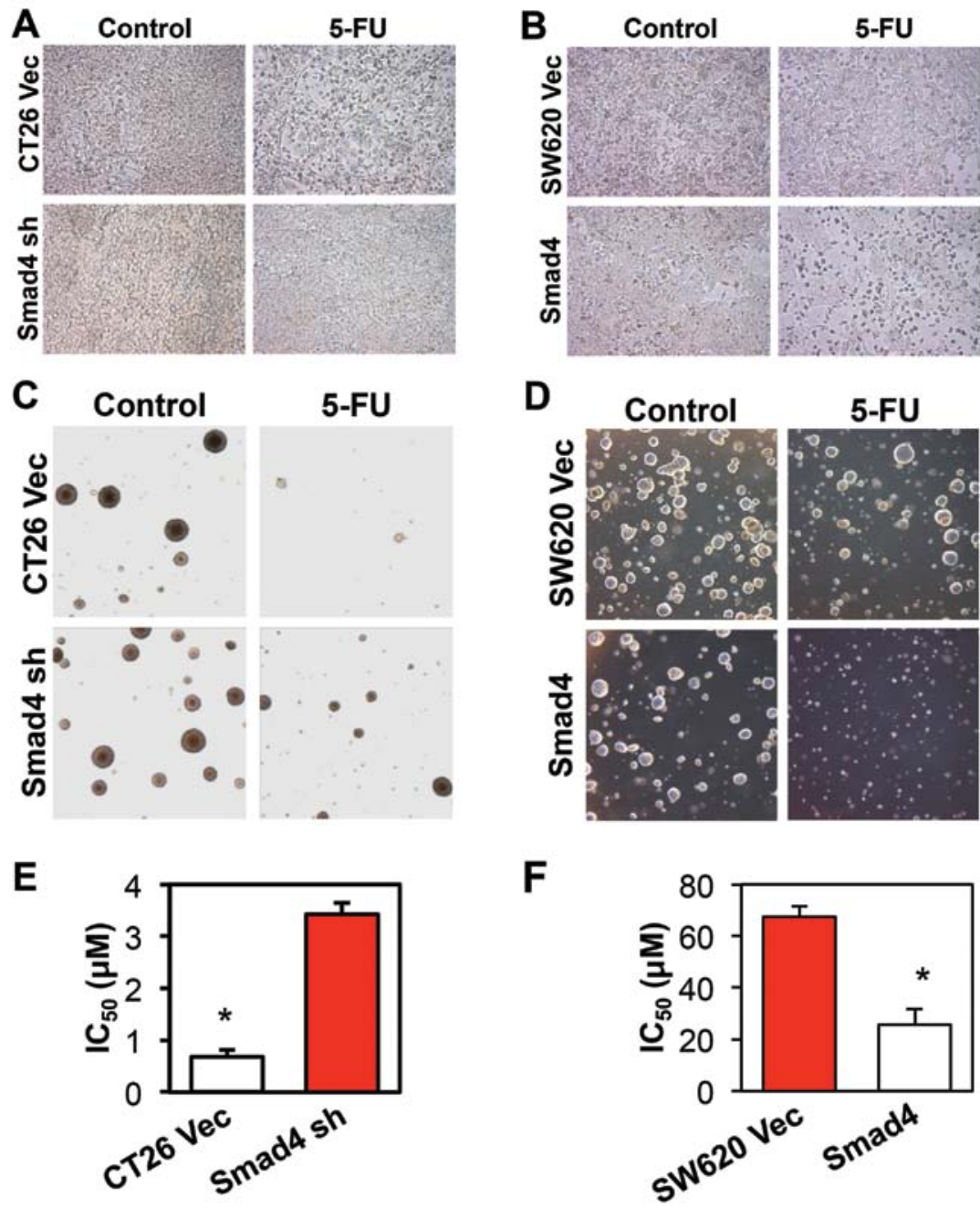

Figure 2. Smad4 promotes the chemosensitivity of CRC cells to 5-FU in vitro. (A) CT26 and (B) SW620 cells were cultured in 6-well plates. 5-FU treatment was initiated once the cells were $30 \%$ confluent. Images of the cells were captured by phase contrast microscopy $48 \mathrm{~h}$ after treatment of the CT26 cells, and $72 \mathrm{~h}$ after treatment of the SW620 cells. (C) CT26 and (D) SW620 cells were grown in 4\% soft agarose, and 5-FU treatment was initiated $48 \mathrm{~h}$ after splitting. Images were captured of the growing colonies. (E) CT26 and (F) SW620 cells were cultured in microtiter plates and incubated overnight. Cells were treated with different concentrations of 5-FU for $72 \mathrm{~h}$. The number of viable cells was determined by the MTT assay. $\mathrm{IC}_{50}$ values were calculated using linear or logarithmic regression $\left(\mathrm{R}^{2}>0.9\right)$. Values are presented as the mean $\pm \mathrm{SD}$ from three independent experiments. ${ }^{\mathrm{P}} \mathrm{P}<0.001$.

with our previous study, Akt was activated in the Smad4 knockdown CT26 and Smad4-null SW620 cells. Moreover, ERK was also activated in the Smad4-deficient cells (Fig. 4A). As 5-FU is a cell cycle-specific drug, we next tried to investigate whether the Akt or ERK pathway modified by Smad4 affects cell cycle-related proteins.

CDC2 (CDK1) is a cyclin-dependent protein kinase, which regulates the G2-M mitotic cell cycle checkpoint through the CDC2/cyclin B complex (15). In addition, the CDC2/cyclin B complex regulates survivin, which inhibits apoptosis and also regulates the cell cycle $(16,17)$. In the present study, we observed that CDC2 and cyclin B were amplified in the CT26 cells when Smad4 was knockdown, while they were reduced in the SW620 Smad4-overexpressing cells (Fig. 4B).

Cell cycle distribution was next examined by flow cytometric analysis. We observed that Smad4 in the CRC cells triggered cell cycle arrest in both the G1 and G2 phase (Fig. 5A). G1/S and $\mathrm{G} 2 / \mathrm{S}$ ratios indicated that the cell cycle arrest reached significance at the G1 and G2 checkpoint in both the CT26 vector control and SW620 Smad4-overexpressing cells when compared with the Smad4-deficient cells (Fig. 5B).

Smad4 promotes $5-F U$ sensitivity through $G 2 / M$ cell cycle arrest and apoptosis by inhibition of the PI3K/Akt/CDC2/ survivin pathway. As both cell cycle arrest and cell apoptosis altered by CDC2 expression may affect drug sensitivity, this result led us to hypothesis that CDC2 amplification in Smad4 deficient/null cells may contribute to 5-FU resistance.

We then examined the $\mathrm{CDC} 2 /$ cyclin B/survivin cascade with 5-FU treatment in the two model cell lines. In the CT26 Smad4-deficient cells, CDC2 expression was significantly increased $24 \mathrm{~h}$ after 5-FU treatment, and thereafter was reversed to a lower level after a 48 -h treatment. In contrast, CDC2 expression in the CT26 vector control cells was continuously downregulated after a 24-h treatment with 5-FU. In this cell line, the cyclin B1 and survivin expression levels were strictly correlated with CDC2 expression (Fig. 6A). Consistent with this result, 5-FU treatment downregulated 

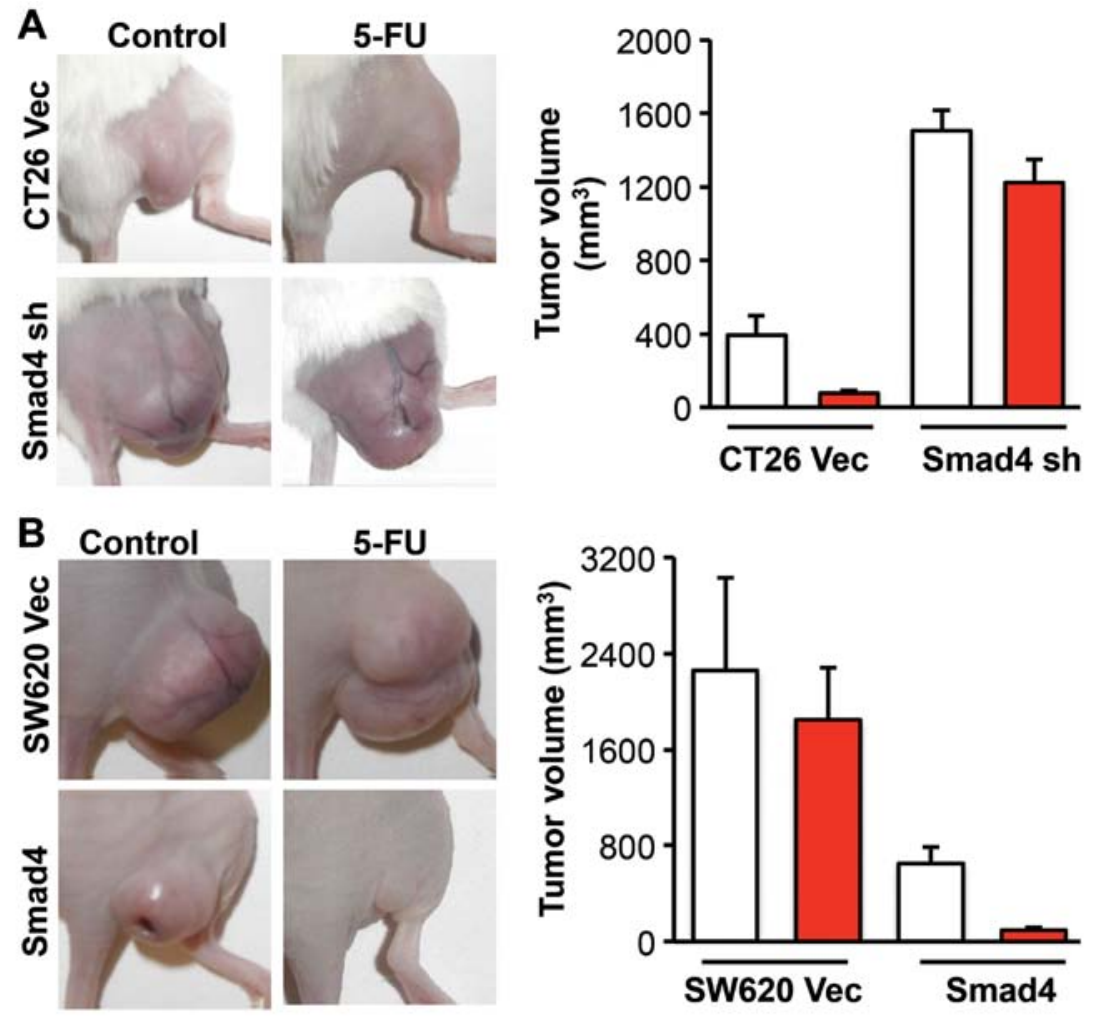

Figure 3. Smad4 promotes the chemosensitivity of CRC to 5-FU in a mouse model. (A) CT26 Smad4-knockdown cells (Smad4 sh) and (B) SW620 Smad4overexpressing cells (Smad4) were injected subcutaneously into BALB/c and athymic nude mice, respectively. Images of the tumor masses were captured at the same time point for the mice under anesthesia. One of the mice for both the control and 5-FU treatment groups is shown. The subcutaneous tumors were removed at the end of the experiment, and tumor volume was calculated. Each plot presents the mean \pm SD volume of five mice in each group. Right histograms: red bar, 5-FU-treated tumors; white bar, untreated tumors.

the CDC2/cyclin B1/survivin cascade in the CT26 vector tumors, but not in the CT26 Smad4-deficient tumors in the mouse model (Fig. 6B). In the SW620 vector control cells, 5-FU treatment continuously amplified $\mathrm{CDC} 2$ and survivin expression. CDC2 and survivin were also increased in the SW620 Smad4-transfected cells at 24 and $48 \mathrm{~h}$ following 5-FU treatment, but was decreased following a 72-h treatment with 5-FU (Fig. 6A). However, cyclin B1 which was promoted by 5-FU treatment showed no change between the vector control and the Smad4-overexpressing cells in this cell line. Upregulation of $\mathrm{CDC} 2 /$ cyclin $\mathrm{B}$ and survivin plays a role in promoting cell survival and G2-M cell cycle transition, both of which may contribute to 5-FU resistance. These data indicated that Smad4 inhibited the CDC2/cyclin B/survivin cascade, and thus promoted 5-FU sensitivity through promotion of cell apoptosis and G2-M cell cycle arrest.

To elucidate the mechanism of Smad4 in regulating the $\mathrm{CDC} 2 /$ cyclin $\mathrm{B} /$ survivin cascade, we next focused on the PI3K/Akt pathway that plays a pivotal role in cell survival and cell cycle regulation. Akt was reported to regulate $\mathrm{CDC} 2$ in different types of malignancies $(18,19)$. In the present study, the expression levels of $\mathrm{CDC} 2$ and survivin were consistently correlated with p-Akt expression in both of the two model cell lines (Fig. 4), which imply that CDC2/survivin is regulated by the Akt pathway. We next used a low dose of the PI3K inhibitor (LY294002) in combination with 5-FU to treat the CT26 and SW620 cells. In the CT26 Smad4-knockdown and SW620 Smad4-null cells, LY294002 significantly inhibited CDC2 and

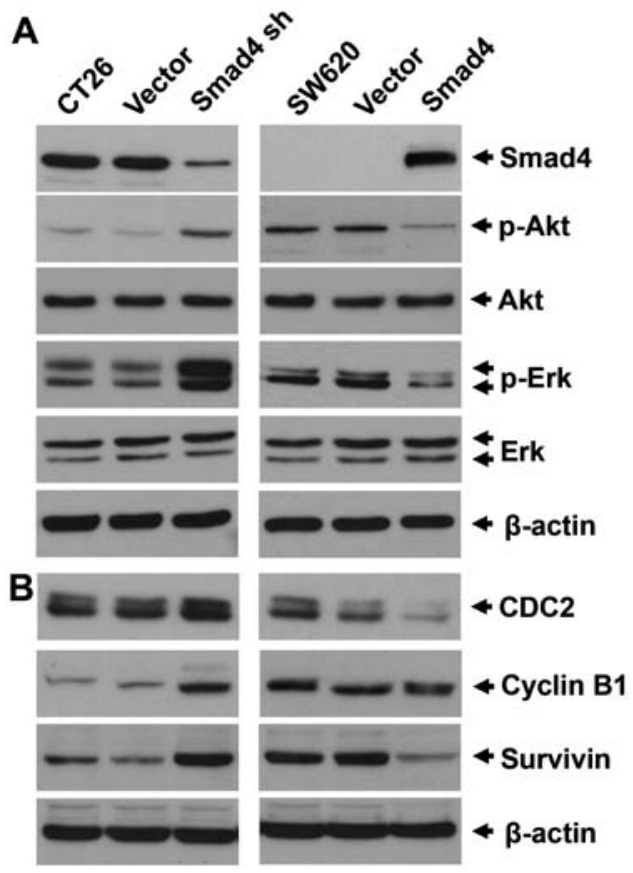

Figure 4. Smad4 deficiency in CRC activates Akt and ERK and regulates cell cycle-related proteins. Lysates from the different CT26 and SW620 cells were used for western blotting to examine (A) Smad4, p-Akt/Akt, p-ERK/ ERK, (B) CDC2, cyclin B1 and survivin.

cyclin B expression under 5-FU treatment, while a lower effect was noted in these cells without 5-FU treatment (Fig. 6C). 

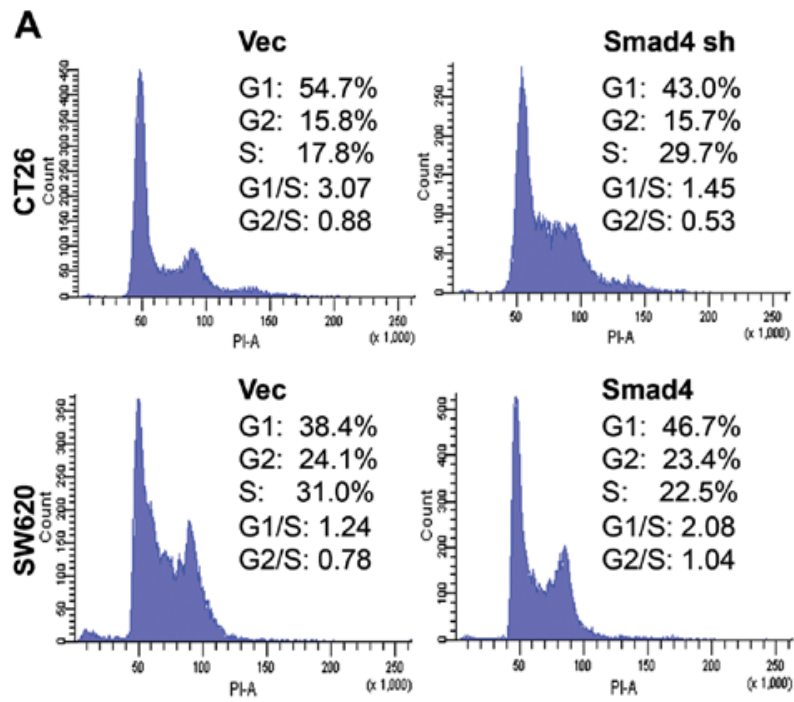
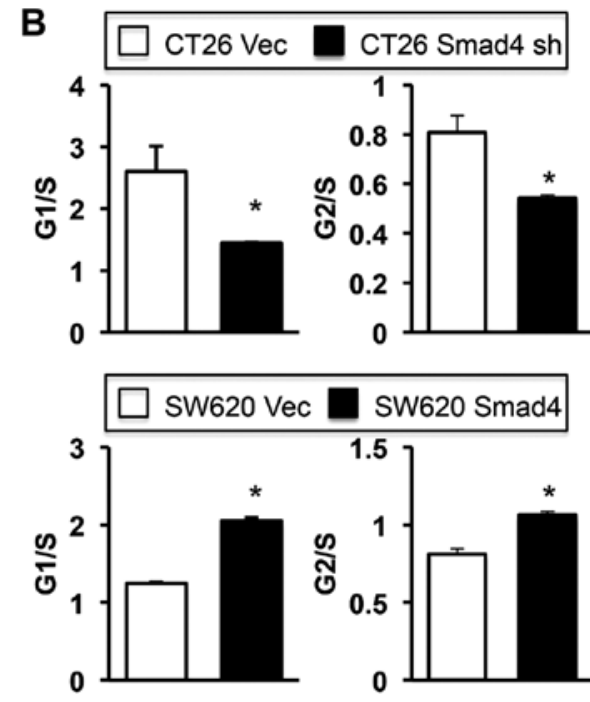

Figure 5. Smad4 deficiency in CRC attenuates cell cycle arrest in the G1 and G2 phase. (A) CT26 and SW620 cells were assessed by flow cytometry for cell cycle distribution. (B) G1/S and G2/S ratios were analyzed. Each bar presents the mean $\pm \mathrm{SD}$ of three independent experiments in each group. $\mathrm{P}<0.01$.

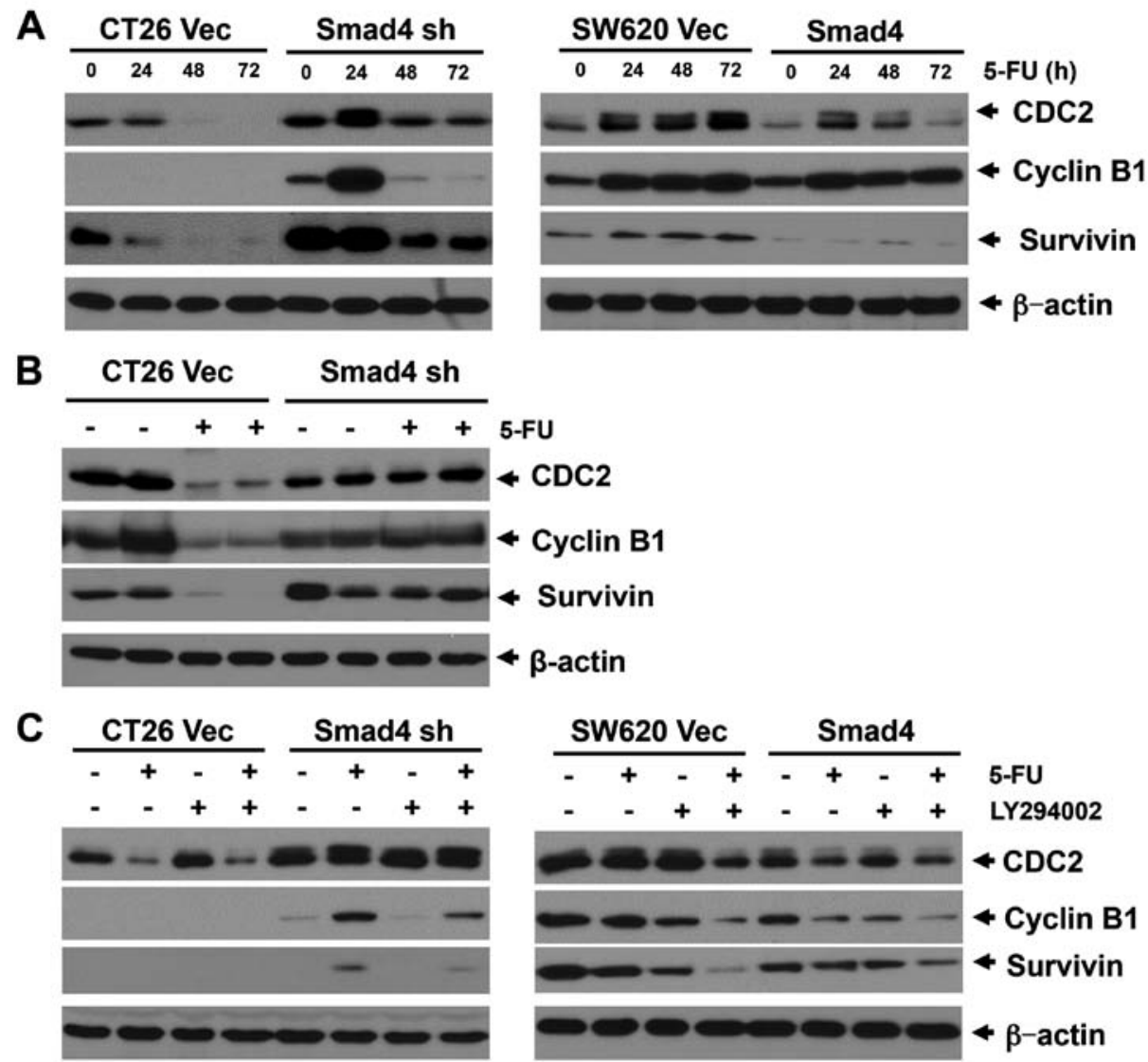

Figure 6. Smad4 promotes 5-FU sensitivity through G2/M cell cycle arrest by inhibition of the PI3K/Akt/CDC2/survivin pathway. (A) Western blot assay. CT26 and SW620 cells were treated with 5-FU once the cells were 30\% confluent. Cell lysate were generated at different time points as indicated after treatment. Total protein was used to check CDC2, cyclin B1 and survivin. (B) Western blotting. Subcutaneous tumor lysates generated from the mice in Fig. 3A were used for western blotting, and CDC2, cyclin B1 and survivin were examined. Results of two representative tumors from each group are shown. (C) CT26 and SW620 cells were treated with 5-FU and/or LY294002 for $24 \mathrm{~h}$, and cell lysates were used for western blot analyses.

In contrast, LY294002 showed a slight effect on CDC2 and cyclin B expression in the Smad4 expressed CT26 cells or Smad4 overexpressed SW620 cells. In addition, we previously reported that LY294002 had a stronger effect on inhibiting survivin expression in Smad4-deficient cells under 5-FU treatment, when compared with CT26 or SW620 cells with higher 


\section{A}
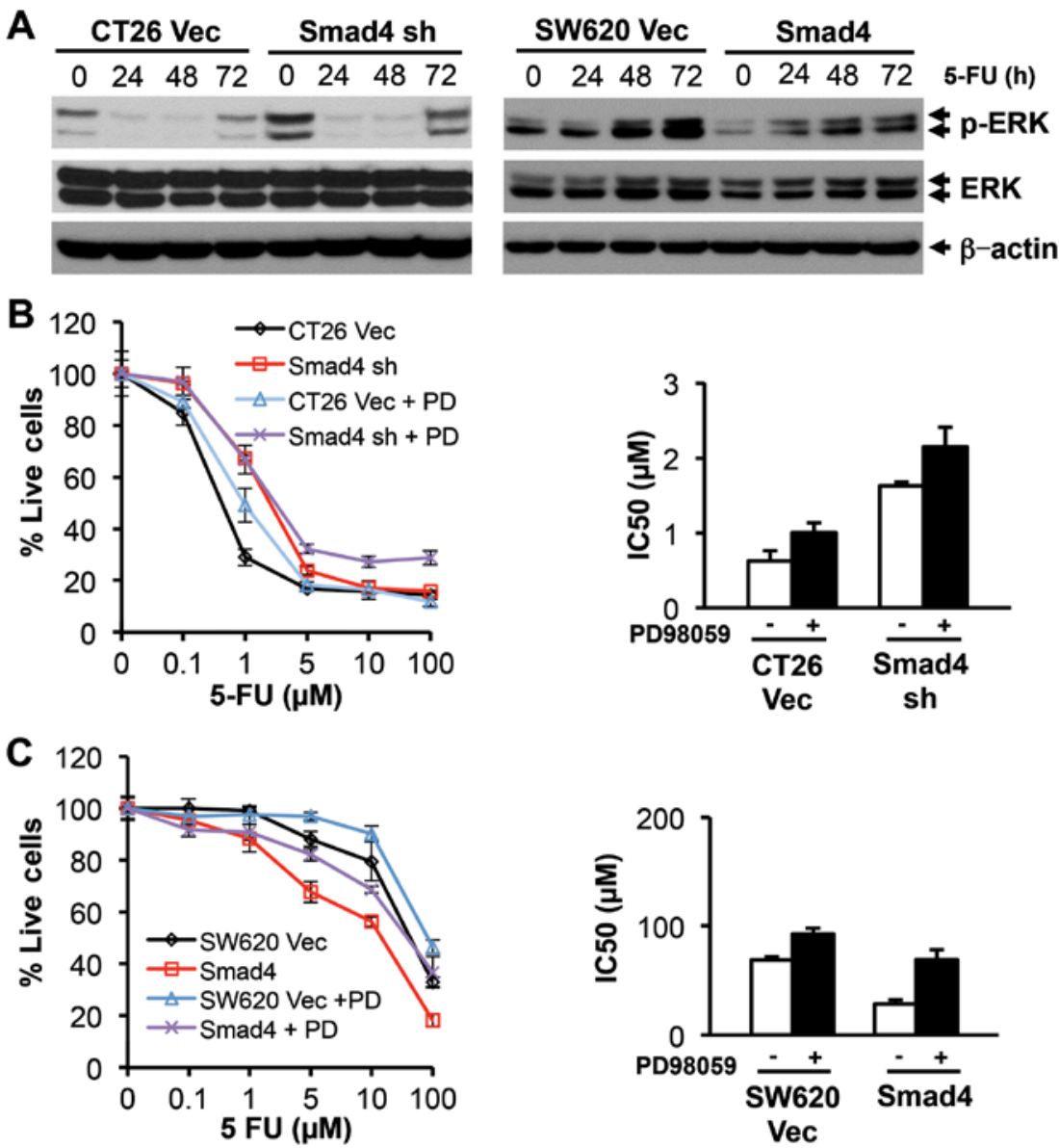
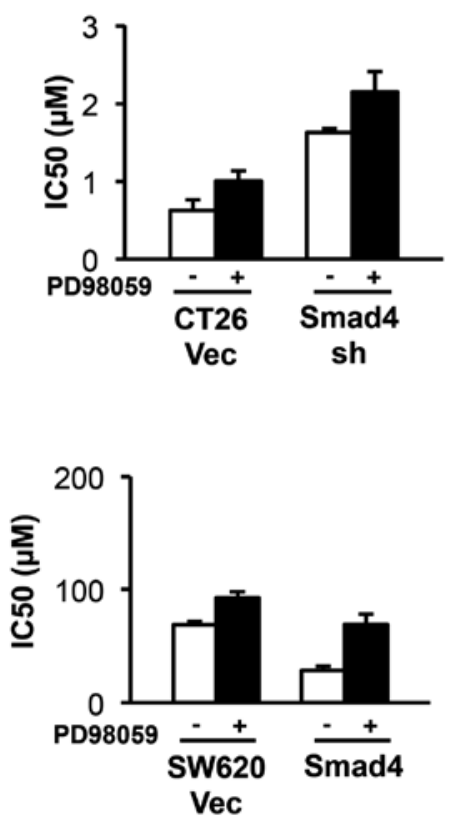

Figure 7. MEK/ERK pathway contributes little to Smad4-induced chemosensitivity in CRC. (A) Western blot assay. CT26 and SW620 cells were treated with 5-FU. Cell lysates were generated at different time points as indicated after treatment. Total protein was used to check p-ERK/ERK. (B) CT26 and (C) SW620 cells growing in microtiter plates were pretreated with MEK1/2 inhibitor PD98059 (PD) and the indicated concentrations of 5-FU for $72 \mathrm{~h}$. The number of viable cells was determined by the MTT assay, and relative cell growth was calculated by comparison to the cells without 5-FU treatment. The chemosensitivity assay was assessed in triplicate. Data are presented as mean \pm SD.

Smad4 expression (9). This evidence indicates that LY294002 has a synergistic effect on promoting 5-FU-induced apoptosis, through inhibition of the $\mathrm{CDC} 2 /$ cyclin $\mathrm{B} /$ survivin cell survival pathway.

Taken together, these results indicated that the inhibition of Smad4 by the PI3K/Akt/CDC2/survivin pathway contributes to cell cycle arrest, thereby restoring 5-FU sensitivity in CRC.

MEK/ERK pathway contributes little to Smad4-induced chemosensitivity in CRC. The ERK pathway is a controversial factor that may effect 5-FU sensitivity. In the different cell lines, the ERK pathway was reported either required or resistant to 5-FU-induced apoptosis (20). In CT26 cells, p-ERK was activated in the Smad4-deficient clones compared with the vector control, and was depressed following 5-FU treatment in both vector control and Smad4-knockdown clones (Fig. 7A). In the SW620 cells, p-ERK was downregulated in the Smad4-overexpressing clones, and was increased in a time-dependent manner following 5-FU treatment in both the control and Smad4-transfected clones (Fig. 7A). We next used MEK inhibitor, PD98059, to elucidate the role of ERK on 5-FU sensitivity in these two cell lines. PD98059 treatment made both CT26 and SW620 cells less sensitive to 5-FU treatment (Fig. 7B and C), but this effect was not in a Smad4-dependent manner. In addition, inhibition of the MEK/ERK pathway did not affect protein expression of either the anti-apoptotic Bcl2 family or pro-apoptotic Bim and Bad (data not shown). These results indicate that the ERK pathway contributes little to Smad4-induced chemosensitivity in either the CT26 or SW620 cells.

\section{Discussion}

TGF- $\beta$ signaling plays a bilateral role in CRC progression and prognosis, depending on the condition of the intracellular Smad and non-Smad pathway. Smad4, a pivotal signal of the TGF- $\beta$ Smad pathway, acts as a tumor-suppressor gene in CRC via interruption of the TGF- $\beta$ signaling pathway (21). This gene was reported to predict the sensitivity to chemotherapy and prognosis in CRC in several clinical studies $(2-6,8)$. Our present research provides evidence that Smad4 expression was reduced with the advance of tumor stages (Fig. 1), which was consistent with previous clinical studies. In the present study, we used two CRC cell lines: a murine CT26 cell line with Smad4 expression sensitive to 5-FU and a human Smad4-null SW620 cell line resistant to 5-FU. Smad4 deficiency in CRC 
cells promoted tumor progression. This evidence is consistent with our previous results (10) and a recent study (22). In addition, we showed for the first time in the present study that Smad4 sensitizes CRC to chemotherapy through downregulation of the PI3K/Akt/CDC2/survivin cascade.

It is believed that inactivation of the Smad pathway coupled with activation of the non-Smad pathway is essential to reverse TGF- $\beta$ from a tumor suppressor to a tumor promoter. Therefore, crosstalk between Smad and the non-Smad pathway plays a critical role in regulating tumor progression, although the exact mechanism of this crosstalk remains elusive. We previously showed that Smad4 overexpression reverses TGF- $\beta$ from a tumor promoter to a tumor suppressor (10). In the present study, we identified that Smad4 deficiency in CT26 cells induced tumor progression in vivo and in vitro. In both cell line models, TGF- $\beta$ inhibited cell growth through the Smad pathway while promoted cell growth through the non-Smad pathway. In this sense, knockdown or loss of Smad4 in these two cell lines promoted tumor progression through inhibition of the tumor-suppressive activity of TGF- $\beta$ coupled with activation of the tumor-promotor function of the non-Smad pathway.

5-FU is the most commonly used chemotherapeutic agent for CRC. However, resistance to 5-FU-based chemotherapy is still an obstacle owing to great polymorphism in drug metabolizing enzymes, as well as several signaling pathways including TGF- $\beta$ Smad and non-Smad pathway. The combination of agents affecting these pathways with 5-FU-based chemotherapy may improve the effects of therapeutic strategies. In previous clinical studies, low Smad4 expression in CRC predicted less sensitivity to 5-FU-based chemotherapy and poor prognosis $(2-6,8)$. Our animal studies showed a consistent observation (Fig. 3). The present study also showed that Smad4 expression was reduced with tumor progression. In the experimental study, we showed that Smad4 had an effect on inducing 5-FU chemosensitivity both in vitro and in vivo, which was consistent with the clinical results.

Although increasing evidence shows the role of Smad4 in 5-FU chemoresistance, the mechanism remains unknown. To the best of our knowledge, there is little research regarding the mechanism of Smad4 in inducing chemosensitivity. To reveal the mechanism, we focused on the non-Smad signals which were regulated by Smad4 expression, as several nonSmad signals contribute to chemosensitivity, with the MEK/ ERK and PI3K/Akt pathway being well elucidated (23-29). There is much controversy regarding the effect of the MEK/ ERK pathway on chemotherapeutic drug-induced apoptosis. This pathway was reported to be either required (30-32) or resistant (28) to chemotherapy, depending on the cell types and genetic background. In the present study, the MEK/ERK pathway was required for 5-FU-induced apoptosis in the CT26 and SW620 cells, but it did not contribute to Smad4-induced chemosensitivity. We finally observed that Smad4 promoted chemosensitivity through the inhibition of the PI3K/Akt pathway (Fig. 4).

Akt activation upregulates $\mathrm{CDC} 2$, which in combination with cyclin B is necessary for G2-M cell cycle transition. CDC2 upregulates the anti-apoptotic factor survivin, which inhibits caspase-dependent apoptosis, therefore promoting cell survival $(16,17)$. Inhibition of the CDC2 and cyclin B complex led to G2-M cell cycle arrest, and genetic knockout of CDC2 or survivin was reported to result in embryo lethality (15). Survivin has been reported to be a highly differentially expressed gene in different types of human cancers including CRC (33), when compared with corresponding normal tissues. The role of the Akt/CDC2/survivin pathway in regulating cell cycle checkpoint and apoptosis makes it crucial for cell sensitivity to drug treatment. Our results obtained by evaluation of cell cycle kinetics revealed that cells with high Smad4 expression were arrested in the $\mathrm{G} 2-\mathrm{M}$ phase under $5-\mathrm{FU}$ treatment, owing to inhibition of the $\mathrm{CDC} 2 /$ cyclin $\mathrm{B} /$ survivin cascade. Accumulation of cells in the G1/S phase may result in resistance to 5-FU treatment, which is consistent with previous studies (34-36). Smad4 reduced the CDC2/cyclin B complex and survivin in both the CT26 and SW620 cells, through the downregulation of the PI3K/Akt pathway, resulting in cell cycle arrest and cell apoptosis. Thus, we believe that Smad4 sensitizes CT26 and SW620 cells to 5-FU treatment, at least partially, through inhibition of the Akt/CDC2/survivin pathway. Either the anti-apoptotic effect or cell cycle checkpoint regulation of this pathway may contribute to 5-FU sensitivity.

In summary, the present study suggests that Smad4 induces chemosensitivity both in murine CT26 and human SW620 cells, through G1 or G2 cell cycle arrest by inhibiting the PI3K/Akt/CDC2/survivin cascade. LY294002 reversed the chemosensitivity of CRC with low Smad4 expression by inhibiting the PI3K/Akt/CDC2/survivin cascade. Smad4 may be a candidate biomarker for the combined use of LY294002 and 5-FU-based chemotherapy for patients with CRC.

\section{Acknowledgements}

The present study was supported by the State Key Project on Infectional Disease of China (grant no. 2012ZX10002016-004 and 2012ZX10002010-001-004), the Chinese Ministry of Public Health for Key Clinical Projects (no. 439, 2010) to Dr Xiaoping Chen and the National Nature Science Foundation of China (no. 81502524) to Dr Binhao Zhang.

\section{References}

1. Siegel R, Naishadham D and Jemal A: Cancer statistics, 2013. CA Cancer J Clin 63: 11-30, 2013.

2. Boulay JL, Mild G, Lowy A, Reuter J, Lagrange M, Terracciano L, Laffer U, Herrmann R and Rochlitz C: SMAD4 is a predictive marker for 5-fluorouracil-based chemotherapy in patients with colorectal cancer. Br J Cancer 87: 630-634, 2002.

3. Alhopuro P, Alazzouzi H, Sammalkorpi H, Dávalos V, Salovaara R, Hemminki A, Järvinen H, Mecklin JP, Schwartz S Jr, Aaltonen LA, et al: SMAD4 levels and response to 5-fluorouracil in colorectal cancer. Clin Cancer Res 11: 6311-6316, 2005.

4. Ahn BK, Jang SH, Paik SS and Lee KH: Smad4 may help to identify a subset of colorectal cancer patients with early recurrence after curative therapy. Hepatogastroenterology 58: 1933-1936, 2011.

5. Kawakami M, Yamaguchi T, Takahashi K, Matsumoto H, Yasutome M, Horiguchi S, Hayashi Y, Funata N and Mori T: Assessment of SMAD4, p53, and Ki-67 alterations as a predictor of liver metastasis in human colorectal cancer. Surg Today 40: 245-250, 2010.

6. Baraniskin A, Munding J, Schulmann K, Meier D, Porschen R, Arkenau HT, Graeven U, Schmiegel W, Tannapfel A and Reinacher-Schick A: Prognostic value of reduced SMAD4 expression in patients with metastatic colorectal cancer under oxaliplatin-containing chemotherapy: A translational study of the AIO Colorectal Study Group. Clin Colorectal Cancer 10: 24-29, 2011. 
7. Papageorgis $\mathrm{P}$, Cheng K, Ozturk S, Gong Y, Lambert AW, Abdolmaleky HM, Zhou JR and Thiagalingam S: Smad4 inactivation promotes malignancy and drug resistance of colon cancer. Cancer Res 71: 998-1008, 2011.

8. Roth AD, Delorenzi M, Tejpar S, Yan P, Klingbiel D, Fiocca R, d'Ario G, Cisar L, Labianca R, Cunningham D, et al: Integrated analysis of molecular and clinical prognostic factors in stage II/III colon cancer. J Natl Cancer Inst 104: 1635-1646, 2012.

9. Zhang B, Zhang B, Chen X, Bae S, Singh K, Washington MK and Datta PK: Loss of Smad4 in colorectal cancer induces resistance to 5-fluorouracil through activating Akt pathway. $\mathrm{Br} \mathrm{J}$ Cancer 110: 946-957, 2014.

10. Zhang B, Halder SK, Kashikar ND, Cho YJ, Datta A, Gorden DL, Datta PK: Antimetastatic role of Smad4 signaling in colorectal cancer. Gastroenterology 138: 969.e1-3-980.e1-3, 2010.

11. Halder SK, Beauchamp RD and Datta PK: Smad7 induces tumorigenicity by blocking TGF-beta-induced growth inhibition and apoptosis. Exp Cell Res 307: 231-246, 2005.

12. Halder SK, Beauchamp RD and Datta PK: A specific inhibitor of TGF-beta receptor kinase, SB-431542, as a potent antitumor agent for human cancers. Neoplasia 7: 509-521, 2005

13. Fanciullino R, Giacometti S, Mercier C, Aubert C, Blanquicett C, Piccerelle $\mathrm{P}$ and Ciccolini J: In vitro and in vivo reversal of resistance to 5-fluorouracil in colorectal cancer cells with a novel stealth double-liposomal formulation. Br J Cancer 97: 919-926, 2007.

14. Wagner M, Roh V, Strehlen M, Laemmle A, Stroka D, Egger B, Trochsler M, Hunt KK, Candinas D and Vorburger SA: Effective treatment of advanced colorectal cancer by rapamycin and 5-FU/oxaliplatin monitored by TIMP-1. J Gastrointest Surg 13: 1781-1790, 2009.

15. Malumbres $\mathrm{M}$ and Barbacid M: Cell cycle, CDKs and cancer: A changing paradigm. Nat Rev Cancer 9: 153-166, 2009.

16. O'Connor DS, Wall NR, Porter AC and Altieri DC: A p34 ${ }^{\mathrm{cdc} 2}$ survival checkpoint in cancer. Cancer Cell 2: 43-54, 2002.

17. O'Connor DS, Grossman D, Plescia J, Li F, Zhang H, Villa A, Tognin S, Marchisio PC and Altieri DC: Regulation of apoptosis at cell division by $\mathrm{p} 34^{\mathrm{cdc} 2}$ phosphorylation of survivin. Proc Natl Acad Sci USA 97: 13103-13107, 2000.

18. Chen Q, Li W, Wan Y, Xia X, Wu Q, Chen Y, Lai Z, Yu C and Li W: Amplified in breast cancer 1 enhances human cholangiocarcinoma growth and chemoresistance by simultaneous activation of Akt and Nrf2 pathways. Hepatology 55: 1820-1829, 2012.

19. Chao JI, Su WC and Liu HF: Baicalein induces cancer cell death and proliferation retardation by the inhibition of CDC2 kinase and survivin associated with opposite role of p38 mitogen-activated protein kinase and AKT. Mol Cancer Ther 6: 3039-3048, 2007.

20. McCubrey JA, Steelman LS, Chappell WH, Abrams SL, Wong EW, Chang F, Lehmann B, Terrian DM, Milella M, Tafuri A, et al: Roles of the Raf/MEK/ERK pathway in cell growth, malignant transformation and drug resistance. Biochim Biophys Acta 1773: 1263-1284, 2007.

21. Cancer Genome Atlas N; Cancer Genome Atlas Network: Comprehensive molecular characterization of human colon and rectal cancer. Nature 487: 330-337, 2012.

22. Freeman TJ, Smith JJ, Chen X, Washington MK, Roland JT, Means AL, Eschrich SA, Yeatman TJ, Deane NG and Beauchamp RD: Smad4-mediated signaling inhibits intestinal neoplasia by inhibiting expression of $\beta$-catenin. Gastroenterology 142: 562-571 e562, 2012
23. Kodach LL, Bos CL, Durán N, Peppelenbosch MP, Ferreira CV and Hardwick JC: Violacein synergistically increases 5-fluorouracil cytotoxicity, induces apoptosis and inhibits Akt-mediated signal transduction in human colorectal cancer cells. Carcinogenesis 27: 508-516, 2006.

24. Jeung HC, Che XF, Haraguchi M, Zhao HY, Furukawa T, Gotanda T, Zheng CL, Tsuneyoshi K, Sumizawa T, Roh JK, et al: Protection against DNA damage-induced apoptosis by the angiogenic factor thymidine phosphorylase. FEBS Lett 580: 1294-1302, 2006.

25. Cantley LC: The phosphoinositide 3-kinase pathway. Science 296: $1655-1657,2002$.

26. Zheng G, Xiong Y, Yi S, Zhang W, Peng B, Zhang Q and He Z: 14-3-3 $\sigma$ regulation by $\mathrm{p} 53$ mediates a chemotherapy response to 5-fluorouracil in MCF-7 breast cancer cells via Akt inactivation. FEBS Lett 586: 163-168, 2012

27. Knuefermann C, Lu Y, Liu B, Jin W, Liang K, Wu L, Schmidt M, Mills GB, Mendelsohn J and Fan Z: HER2/PI-3K/Akt activation leads to a multidrug resistance in human breast adenocarcinoma cells. Oncogene 22: 3205-3212, 2003.

28. Jin W, Wu L, Liang K, Liu B, Lu Y and Fan Z: Roles of the PI-3K and MEK pathways in Ras-mediated chemoresistance in breast cancer cells. Br J Cancer 89: 185-191, 2003.

29. Taiyoh H, Kubota T, Fujiwara H, Matsumura A, Murayama Y, Okamoto K, Ichikawa D, Ochiai T, Nakamura T, Matsumoto K, et al: NK4 gene expression enhances 5-fluorouracil-induced apoptosis of murine colon cancer cells. Anticancer Res 31: 2217-2224, 2011

30. Wang X, Martindale JL and Holbrook NJ: Requirement for ERK activation in cisplatin-induced apoptosis. J Biol Chem 275 39435-39443, 2000.

31. Woessmann W, Chen X and Borkhardt A: Ras-mediated activation of ERK by cisplatin induces cell death independently of p53 in osteosarcoma and neuroblastoma cell lines. Cancer Chemother Pharmacol 50: 397-404, 2002.

32. Basu A and Tu H: Activation of ERK during DNA damageinduced apoptosis involves protein kinase Cdelta. Biochem Biophys Res Commun 334: 1068-1073, 2005.

33. Hernandez JM, Farma JM, Coppola D, Hakam A, Fulp WJ, Chen DT, Siegel EM, Yeatman TJ and Shibata D: Expression of the antiapoptotic protein survivin in colon cancer. Clin Colorectal Cancer 10: 188-193, 2011.

34. Noda T, Nagano H, Takemasa I, Yoshioka S, Murakami M, Wada H, Kobayashi S, Marubashi S, Takeda Y, Dono K, et al: Activation of Wnt/beta-catenin signalling pathway induces chemoresistance to interferon-alpha/5-fluorouracil combination therapy for hepatocellular carcinoma. Br J Cancer 100: $1647-1658,2009$

35. Guo X, Goessl E, Jin G, Collie-Duguid ES, Cassidy J, Wang W and O'Brien V: Cell cycle perturbation and acquired 5-fluorouracil chemoresistance. Anticancer Res 28: 9-14, 2008.

36. Gavin EJ, Song B, Wang Y, Xi Y and Ju J: Reduction of Orc6 expression sensitizes human colon cancer cells to 5-fluorouracil and cisplatin. PLoS One 3: e4054, 2008. 